\title{
Long noncoding RNA MIR31HG and its splice variants regulate proliferation and migration: prognostic implications for muscle invasive bladder cancer
}

Sheng Wu ${ }^{1,2}$, Katja Nitschke ${ }^{1}$, Thomas Stefan Worst ${ }^{1}$, Alexander Fierek ${ }^{1}$, Cleo-Aron Weis ${ }^{3}$, Markus Eckstein ${ }^{4}$, Stefan Porubsky ${ }^{3}$, Maximilian Kriegmair ${ }^{1}$ and Philipp Erben ${ }^{1 *}$

\begin{abstract}
Background: Growing evidence supports the pivotal role of long non-coding RNAs (IncRNAs) in the regulation of cancer development and progression. Their expression patterns and biological function in muscle invasive bladder cancer (MIBC) remain elusive.

Methods: Transcript levels of IncRNA miR-31 host gene (MIR31HG) and its splice variants were measured in our MIBC cohort $(n=102)$ by qRT-PCR, and validated in silico by the TCGA cohort $(n=370)$. Kaplan-Meier and multiple Cox regression analysis were conducted to evaluate the survival significance of MIR31HG and its splice variants. Functional experiments were performed to examine the proliferation and migration abilities of MIR31HG and its splice variants by knockdown approaches.

Results: In this study, a decreased expression of MIR31HG was found in bladder cancer cells and tissues, except in the basal subtype. Survival analysis showed that high expression of MIR31HG was associated with poor overall survival (OS) and disease-free survival (DFS) in patients with MIBC of basal subtype. Two splice variants of MIR37HG lacking exon 1 (MIR31HGDE1) and exon 3 (MIR31HG $E$ E3) were identified to have specific expression patterns in different molecular subtypes of our MIBC cohort. MIR31HG $\triangle E 3$ was highly expressed in basal subtype tumors. A high expression of MIR31HG $\triangle E 1$ and MIR31HG $E E 3$ was associated with worse OS and DFS in our cohort. In vitro experiments revealed that knockdown of MIR37HG inhibits cell proliferation, colony formation, and migration in bladder cancer. Cell proliferation and migration assays after knockdown of splice variants of MIR31HG showed corresponding roles for the full-length transcript.
\end{abstract}

Conclusions: Our study demonstrates that MIR3THG and its splice variants could serve as biomarkers for the classification and prognosis prediction of patients with MIBC.

Keywords: LncRNA, MIR37HG, Muscle invasive bladder cancer, Biomarker, Molecular subtype

\footnotetext{
* Correspondence: philipp.erben@medma.uni-heidelberg.de

'Department of Urology and Urosurgery, Medical Faculty Mannheim, University of Heidelberg, 68167 Mannheim, Germany

Full list of author information is available at the end of the article
}

(c) The Author(s). 2020 Open Access This article is licensed under a Creative Commons Attribution 4.0 International License, which permits use, sharing, adaptation, distribution and reproduction in any medium or format, as long as you give appropriate credit to the original author(s) and the source, provide a link to the Creative Commons licence, and indicate if changes were made. The images or other third party material in this article are included in the article's Creative Commons licence, unless indicated otherwise in a credit line to the material. If material is not included in the article's Creative Commons licence and your intended use is not permitted by statutory regulation or exceeds the permitted use, you will need to obtain permission directly from the copyright holder. To view a copy of this licence, visit http://creativecommons.org/licenses/by/4.0/. The Creative Commons Public Domain Dedication waiver (http://creativecommons.org/publicdomain/zero/1.0/) applies to the data made available in this article, unless otherwise stated in a credit line to the data. 


\section{Background}

Bladder urothelial carcinoma (BLCA) is the tenth most common cancer worldwide with an estimated 550,000 new cases and 200,000 deaths in 2018 [1]. It is widely accepted that BLCA is a heterogeneous disease, which is classified into two distinct subtypes: non-muscle invasive bladder cancer (NMIBC) and muscle invasive bladder cancer (MIBC) [2]. MIBC is responsible for most cases involving metastases leading to death, which arise from 10 to $20 \%$ of advanced NMIBC cases, and its clinical management remains limited [3]. Currently, several classifications have proposed sets of molecular classes, including basal and luminal subtypes, which are partially characterized by KRT5 and KRT20 gene expression [4]. In addition, epidermal growth factor receptor (EGFR) is expressed at high level in basal tumors, involved in controlling the basal gene signature [4]. Although a number of groups have reported molecular classifications of BLCA to evaluate the severity and prognosis of this disease, reliable and effective biomarkers for early diagnosis and prognostic prediction are still lacking $[5,6]$. Thus, in-depth understanding of molecular events and underlying mechanisms involved in the carcinogenesis of MIBC may provide effective therapeutic targets and predictive biomarkers, which are urgently needed.

Long noncoding RNAs (lncRNAs) are a class of RNA transcripts, which are longer than 200 nucleotides and do not have protein-coding capacity [7]. Recent studies have demonstrated that lncRNAs play essential roles in a wide range of biological processes, such as proliferation, apoptosis, cell cycle arrest, cell migration, and invasion $[8,9]$. Furthermore, it has been shown that several lncRNAs are deregulated in many tumors and may be involved in both carcinogenesis and cancer metastasis [10]. Recently, the miR-31 host gene (MIR31HG, also known as LOC554202) has been identified in several cancers, such as breast, colorectal, gastric cancer, and pancreatic ductal adenocarcinoma [11-14]. It is also reported that MIR31HG expression was down-regulated in BLCA cell lines and tumor tissues [15]. However, the functional role of MIR31HG and its association with molecular classifications in BLCA are as yet unknown.

Despite the continuously growing knowledge on lncRNAs and cancer, a reliable clinical molecular marker has not yet been found. Considering that dysregulation of mRNA splicing can trigger cancer signaling pathways and contribute to almost all hallmarks of cancer [16], the alternative splicing of lncRNAs may also impact cellular processes, which could open new possibilities for biomarker discovery. Previously, it was reported that splice variants of osteopontin have prognostic value in breast cancer [17]. In BLCA, CD44 splice variants have been demonstrated to be involved in tumor progression and chemosensitivity [18]. In addition, the lncRNA
PVT1 and its splice variant are highly expressed in clear cell renal cell carcinoma, and function as oncogenic transcripts [19]. These findings advocate for the use of lncRNAs and their splice variants as tissue-specific transcripts and promising prognostic biomarkers in certain cancers. However, the precise function of most lncRNAs and the mechanisms of their molecular regulation remains to be elucidated.

In this study, the expression level and clinicopathologic significance of MIR31HG were first evaluated by in silico database analysis and then by qRT-PCR in a cohort from our institution. Upon knockdown of MIR31HG, a series of in vitro experiments was performed to investigate the effects of MIR31HG on proliferation, colony formation, and migration of BLCA cells. Transcript-specific knockdown with consecutive cell functional assays were performed to determine the role of MIR31HG splice variants and to investigate their association with molecular subtypes of MIBC. Further survival analyses were carried out to determine if MIR31HG and its splice variants could be used as prognostic biomarkers.

\section{Materials and methods}

\section{Patients and tissue samples}

This study retrospectively enrolled 102 patients who received radical cystectomy $(\mathrm{RC})$ at the Department of Urology and Urosurgery of the University Medical Centre Mannheim, between 2008 and 2012, and who had a histological diagnosis of MIBC (males: $n=74,73 \%$, median age: 71 years, range: 41-88 years; females: $n=28,27 \%$, median age: 73 years, range: 47-86 years; Mannheim cohort). All patients were treated with RC and bilateral lymphadenectomy without preoperative chemotherapy or radiotherapy. With the help of the clinic's internal documentation program, the following parameters were collected after examination of the pathology findings: sex, age, T-stage, N-stage, M-stage, grading, lymphovascular invasion (LVI), blood vessel invasion (VI), simultaneous carcinoma in situ (CIS), multifocality, and soft tissue positive surgical margin.

Formalin fixed paraffin embedded (FFPE) tumor tissue samples were evaluated for pathological stage according to the 2017 TNM classification from the Union for International Cancer Control (UICC) [20]. Tumors were graded using the $2017 \mathrm{WHO} / \mathrm{ISUP}$ classification [21]. Studies involving human participants were approved by the ethical board of the University of Heidelberg (2015549 N-MA) and performed in accordance with relevant guidelines and regulations. The Cancer Genome Atlas cohort (TCGA, Provisional) contained RNA sequencing data of 407 patients with MIBC and complete clinicopathological and follow-up data (males: $n=300,73.7 \%$, median age: 68 years, range: 34-90 years; females: $n=$ 107, 26.3\%, median age: 72 years, range: $43-90$ years; 
TCGA cohort). Thirty-seven patients with tumor stage $\mathrm{T} 1$ or not defined $\mathrm{T}$ stage were excluded from the survival analysis $(n=370)$.

\section{Database}

Expression data in transcripts per million (TPM) of $25 \mathrm{hu}-$ man bladder cancer cell lines were collected from the Cancer Cell Line Encyclopedia [22]. Expression of MIR31HG was analyzed by Expression Atlas (https://www.ebi.ac.uk/gxa/ home) and normalized by TPM. Transcript expression data of 370 BLCA samples and 21 normal samples were collected from TCGA (https://tcga-data.nci.nih.gov/tcga/) and analyzed by cBioPortal (http://www.cbioportal.org/), which normalized the samples by reads per kilobase million (RPKM) and $\log 2$ transformation. Expression of transcripts, exons and junctions was collected and analyzed using the Xena online exploration tool (https://xena.ucsc.edu/) and TSVdb (http://www.tsvdb.com) [23]. The IncRNA-protein interactions were analyzed by lncPro [24]. IncPro yields a score using amino acid and nucleotide sequences. This score can be used to measure the interaction between a pair of lncRNA and protein. The sequence of MIR31HG and protein sequences were obtained from the NCBI database (http:// www.ncbi.nlm.nih.gov/).

\section{Cell culture}

In this study six different cell lines were used, including a normal human urothelium cell line (UROtsa), a basallike urothelial carcinoma cell line (SCaBER), two luminal-like urothelial carcinoma cell lines (RT112 and RT4), and two mixed-type urothelial carcinoma cell lines (UMUC3 and T24). UROtsa cells were cultured in Roswell Park Memorial Institute medium (RPMI) supplemented with 5\% fetal bovine serum (FBS). RT112, RT4, SCaBER, and UMUC3 cells were cultured in Dulbecco's modified Eagle's medium (DMEM) containing 10\% FBS. T24 cells were cultured in McCoy's 5A medium containing 10\% FBS. UMUC3, SCaBER, RT112, and T24 cells were obtained from the European Collection of Authenticated Cell Cultures (ECACC), RT4 from the American Type Culture Collection (ATCC), and UROtsa cells from a collaborator. Before starting the experiments, all cell lines were authenticated by Multiplexion (Heidelberg, Germany).

\section{siRNA transfection}

A pool of four different small interfering RNAs (siRNAs) against MIR31HG (designated si-MIR31HG, set of 4), and transcript-specific siRNAs against MIR31HGAE1 and MIR31HGAE3 were transfected into urothelial cells using the DharmaFECT1 siRNA transfection reagent (GE Healthcare Dharmacon, Inc., CO, USA). Scrambled siRNA was used as negative control. All sequences of the siRNAs are listed in Table S1.

\section{Cell viability assay}

Cell viability was analyzed using the 3-(4,5-dimethylthiazol-2-yl)-5-(3-carboxymethoxyphenyl)-2-(4-sulfophenyl)-2H-tetrazolium (MTS) assay (Promega, Wis, USA) according to the manufacturer's instructions with six replicates. MTS solution was added at $0,24,48$, and 72 $\mathrm{h}$ after different siRNA transfections and the absorbance was measured using a microplate reader at $492 \mathrm{~nm}$.

\section{Colony formation assay}

For colony formation assays, urothelial cells were seeded in six-well plates at a concentration of 5000 cells per well in triplicates, and cultured for 7 days before staining viable colonies with crystal violet (Sigma-Aldrich, Darmstadt, Germany). The staining intensity of the colonies was quantitated using ImageJ software [25].

\section{Migration assay}

Cell migration was evaluated by a wound-healing assay. Cells were seeded in cell culture inserts (ibidi $\mathrm{GmbH}$, Munich, Germany) and the open wound area was measured after $12 \mathrm{~h}$. The proportion of the wound area was quantitated using TScratch software [26].

\section{RNA extraction and qRT-PCR}

Total RNA of cells was isolated using RNeasy Mini kits (Qiagen, Hilden, Germany) according to the manufacturer's instructions. For FFPE tissues, RNA was extracted and enriched using the magnetic-bead-based XTRAKT FFPE Kit (Stratifyer, Cologne, Germany) according to the manufacturer's instructions [27]. Next, reverse transcription was performed for cell samples using the MMLV Reverse Transcriptase kit (Thermo Fisher Scientific, Waltham, MA, USA), and for FFPE samples using the Superscript III reverse transcriptase kit (Thermo Fisher Scientific, Waltham, MA, USA) with sequencespecific primers. qPCR was used to measure relative mRNA expression with TaqMan Fast Advanced Master Mix (Thermo Fisher Scientific, Waltham, MA, USA). $\beta$ Glucuronidase (GUS) and calmodulin2 (Calm2) were measured as reference genes [28]. The relative mRNA expression level was normalized to reference genes and determined using the $40-\Delta \mathrm{CT}$ or $2^{-\Delta \Delta \mathrm{CT}}$ method for cell culture samples and FFPE samples, as previously described $[29,30]$. All primers and probes used in this study are shown in Table S2.

\section{Statistical analysis}

Statistical analyses were performed using SPSS 20.0 software (IBM, Chicago, IL, USA) and GraphPad Prism 6.0 (GraphPad Software, La Jolla, CA, USA). A Kolmogorov-Smirnov (K-S) test was used to determine whether the data were normally distributed. Student's t-tests were used to compare groups of normally distributed 
numerical data, while the Mann-Whitney $U$ and Kruskal-Wallis tests were used to compare non-normally distributed numerical data. Linear regression was used to determine the efficiency of amplification. Spearman tests were used to test the correlation between different gene expressions. The cut-off values of the high and low MIR31HG expression groups were determined by receiver operating characteristic (ROC) curve analysis in the Mannheim cohort [31]. Similarly, in the TCGA cohort, the cut-off value ( $\log 2$ value) of high and low MIR31HG expression groups were determined by ROC curve analysis. The Cox regression model was used for univariable and multivariable analysis to calculate hazard ratio (HR). For multivariable analysis, parameters with a cut-off value of $p<0.2$ inform the univariable analysis were included. The expression of KRT5 was used as a marker for basal phenotype. High KRT5 and low KRT5 were defined as expression above and below median. Survival rates of patients were calculated using the Kaplan-Meier method, and comparisons were made with the log-rank test. In all cases, $p<0.05$ was considered statistically significant.

\section{Results}

\section{MIR31HG expression was subtype-specific in bladder} cancer tissues and cell lines

First, in silico analyses of expression of MIR31HG were performed. In the TCGA dataset, the expression of MIR31HG was down-regulated in MIBC (median expression $0.2435, n=370$ ) compared to normal tissues (median expression 0.3549, $n=23, p=0.0002$, Fig. 1a). Patients with BLCA in the TCGA cohort were classified into basal/ squamous, luminal, luminal-infiltrated, luminal-papillary and neuronal subtypes according to mRNA clustering
[32]. Among all subtypes, expression of MIR31HG in basal/squamous (median expression 7.10 with range of 0 to 9.96) subtype was the most abundant, which was higher than in luminal (median expression 3.16 with range of 0.13 to $6.8, p<0.0001$ ), luminal-infiltrated (median expression 4.99 with range of 0 to $9.16, p<0.0001$ ), luminalpapillary (median expression 5.53 with range of 0 to 9.92, $p=0.0002$ ), and neuronal (median expression 4.92 with range of 0 to 8.7, $p=0.0215$ ) subtypes (Fig. 1b). By clustering according to a trichotomous molecular classifications based on an alternative mRNA clustering of the TCGA cohort [33], it is also determined that the expression of MIR31HG was higher in the basal subtype (median expression 7.10 with range of 0 to 9.96) compared to the luminal (median expression 5.21 with range of 0 to 10.16) and infiltrated (median expression 4.99 with range of 0 to 9.16) subtypes (Fig. S1A). Furthermore, due to the significance of lymph node metastasis in MIBC cohort, the expression of MIR31HG in different lymph node status was analyzed. No significant difference was found between lymph node metastasis negative and positive groups $(p=$ 0.2650, Fig. S1B).

Expression of MIR31HG was also detected in a normal urothelial cell line (UROtsa) and three BLCA cell lines (SCaBER, UMUC3 and T24) by qRT-PCR. Significantly lower expression of MIR31HG was observed in UMUC3 (mean expression 33.59, $p=0.0003$ ) and T24 cells (mean expression 32.34, $p<0.0001)$ compared with UROtsa cells (mean expression 34.01). In contrast, expression of MIR31HG was higher in SCaBER cells (mean expression 35.53, $p=0.0200$ ) compared to UROtsa cells. The lowest level of MIR31HG expression was observed in RT112 (mean expression 21.03, $p<0.0001$ ) and RT4 cells (mean expression 23.64, $p<0.0001$, Fig. 1c). Furthermore,

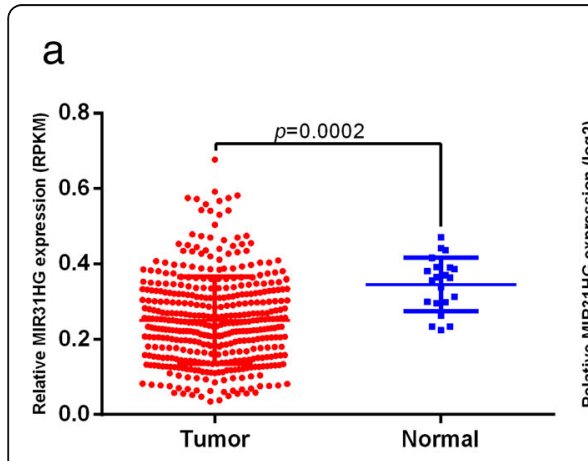

b

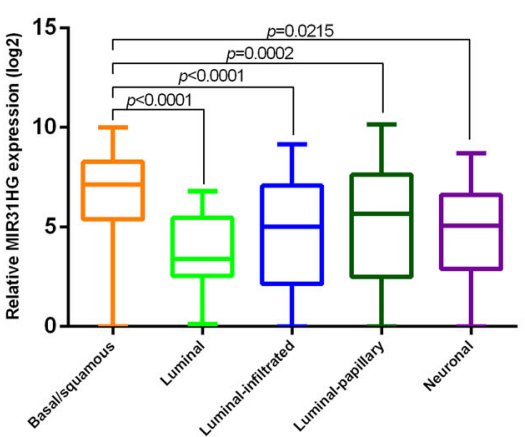

C

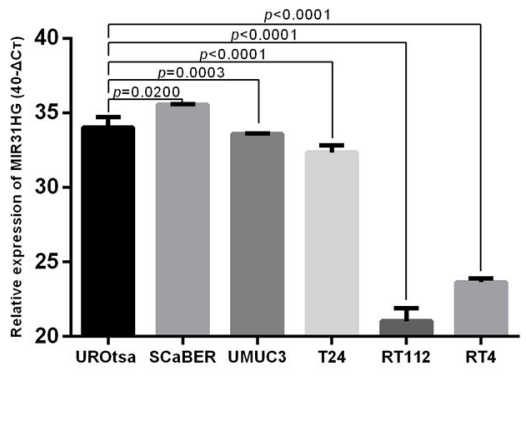

Fig. 1 Expression of MIR31HG in BLCA tissue samples and cell lines. a In the TCGA cohort data, MIR31HG was down-regulated (median expression 0.2435) in tumors compared with normal tissues (median expression 0.3549). b In the TCGA cohort, expression of MIR31HG was higher in basal/squamous (median expression 7.10 with range of 0 to 9.96) subtype compared to luminal (median expression 3.16 with range of 0.13 to 6.8), luminal-infiltrated (median expression 4.99 with range of 0 to 9.16), luminal-papillary (median expression 5.53 with range of 0 to 9.92), and neuronal (median expression 4.92 with range of 0 to 8.7) subtypes. c In a normal urothelial cell line (UROtsa) and five BLCA cell lines (SCaBER, UMUC3, T24, RT112 and RT4), expression of MIR31HG was detected by qPCR. MIR31HG expression was significantly lower in UMUC3 and T24 cells, and higher in SCaBER cells compared to UROtsa cells. The lowest MIR31HG expression was observed in RT112 and RT4 cells 
MIR31HG expression was analyzed in RNA-seq data from the Cancer Cell Line Encyclopedia containing 25 BLCA cell lines, including 20 bladder urothelial cell carcinomas, a bladder squamous cell carcinoma, and four bladder carcinoma cell lines from unknown primaries. The data showed that MIR31HG expression levels in SCaBER cells (expression level: 10 TPM) were higher than in RT112 (expression level: 0 TPM), RT4 (expression level: 0 TPM), and UMUC3 cells (expression level: 3 TPM, Fig. S1C).

\section{Identification and expression of two splice variants of MIR31HG}

Demographic and clinical-pathological data of the 102 patients with MIBC included in the Mannheim cohort are shown in Table 1. Median follow-up of the entire cohort was 21 months (range 3-121 months) and the median follow-up of surviving patients was 50 months (range 9-121 months). In total, 52 patients (50.98\%) suffered a relapse (local relapse $n=6$, lymph nodes and/or distant metastases $n=36$, unclear metastasis pattern $n=$ 10). Of the $56(54.90 \%)$ patients who died during the follow-up, 38 (37.25\%) of them died because of BLCA.

Four transcript variants of MIR31HG were retrieved from the National Center for Biotechnology Information (NCBI) nucleotide database. Transcript variant 1 (RefSeq ID: NR_027054.2) is the full transcript of MIR31HG, containing four exons and three junctions. For simplification, transcript variant 2 (RefSeq ID: NR_152877.1) lacking exon 1 was named MIR31HGDE1, and transcript variant 4 (RefSeq ID: NR_152879.1) lacking exon 3 was named MIR31HGDE3. Transcript-specific primers for each splice variant were designed and are listed in

Table 1 Clinicopathological characteristics of patients and specimens of the Mannheim cohort

\begin{tabular}{ll}
\hline Clinicopathological Features & Number \\
\hline Age & \\
$<70$ & 43 \\
$\geq 70$ & 59 \\
Gender & \\
Male & 74 \\
Female & 28 \\
Stage & \\
T2 & 12 \\
T3 & 69 \\
T4 & 21 \\
Lymph node metastasis & \\
Negative & 70 \\
Positive & 24 \\
Undefined & 8 \\
\hline
\end{tabular}

Supplementary Table 2. A model of the gene sequence of the MIR31HG transcript (ENST00000304425.3) and its two splice variants, MIR31HGAE1 and MIR31HGDE3, is shown in Fig. 2a. In the Mannheim cohort, a significantly higher expression of MIR31HG (median expression 34.7) was found compared to the transcripts, MIR31HGAE1 (median expression 35.5, $p=0.0187$ ) and MIR31HGLE3 (median expression 36.7, $p=0.0001$, Fig. 2b). Expression of MIR31HG junction 3, which reflects MIR31HGAE3 expression, was significant higher in the basal (median expression 0.6267) compared to the luminal (median expression 0.3478) subtype in the TCGA cohort ( $p=0.0081$, Fig. 2c). As the expression of KRT5 and KRT20 can be used as a marker for basal and luminal phenotypes, respectively, the expression of MIR31HG was analyzed in terms of KRT5 and KRT20 dependent expression. High KRT5 (Ct value $>36.55)$ and high KRT20 (Ct value > 34.12) were defined as expression above median. MIR31HGLE1 expression was higher in the high KRT20 group $(p=0.0395$, Fig. $2 d)$, and MIR31HGAE3 expression was higher in the high KRT5 group ( $p=0.1514$, Fig. 2e).

\section{MIR31HG expression was associated with copy-number alterations}

The in silico analysis of MIR31HG copy-number alterations revealed a genetic alteration rate of 25\% (92/370). Putative copy-number alterations including deep/shallow deletion, diploid, gain, and amplification were acquired from the GISTIC (Genomic Identification of Significant Targets in Cancer) algorithm. MIR31HG showed 56.76\% of shallow/deep deletion $(n=210), 31.62 \%$ of diploid $(n=117)$, and $11.62 \%$ of gain/amplification $(n=43$, Fig. 3a). The expression of MIR31HG was significantly higher in the subgroup with gain (median expression 7.46, $p<0.0001$ ) and diploid (median expression 7.12, $p<0.0001)$ compared to those with a deletion alteration (median expression 4.77, Fig. 3b). High expression of MIR31HG was found in the subgroup with deletion and diploid alteration than in gain $(n=47,20.44 \%$ in gain; $n=99,43.04 \%$ in diploid; $n=84,36.52 \%$ in deletion), while low expression was observed in the subgroup with deletion alteration, compared to diploid and gain $(n=$ $15,8.67 \%$ with gain; $n=28,16.18 \%$ with diploid; $n=130$, $75.15 \%$ with deletion, Fig. 3c).

\section{MIR31HG as prognostic marker for patients with basal subtype}

After the analysis of gene expression and copy-number alterations of $M I R 31 H G$, the association with clinical outcome and parameters was analyzed in the TCGA and the Mannheim cohort. The expression of MIR31HG showed no significant correlation with overall survival (OS) and disease free survival (DFS) in the full TCGA 


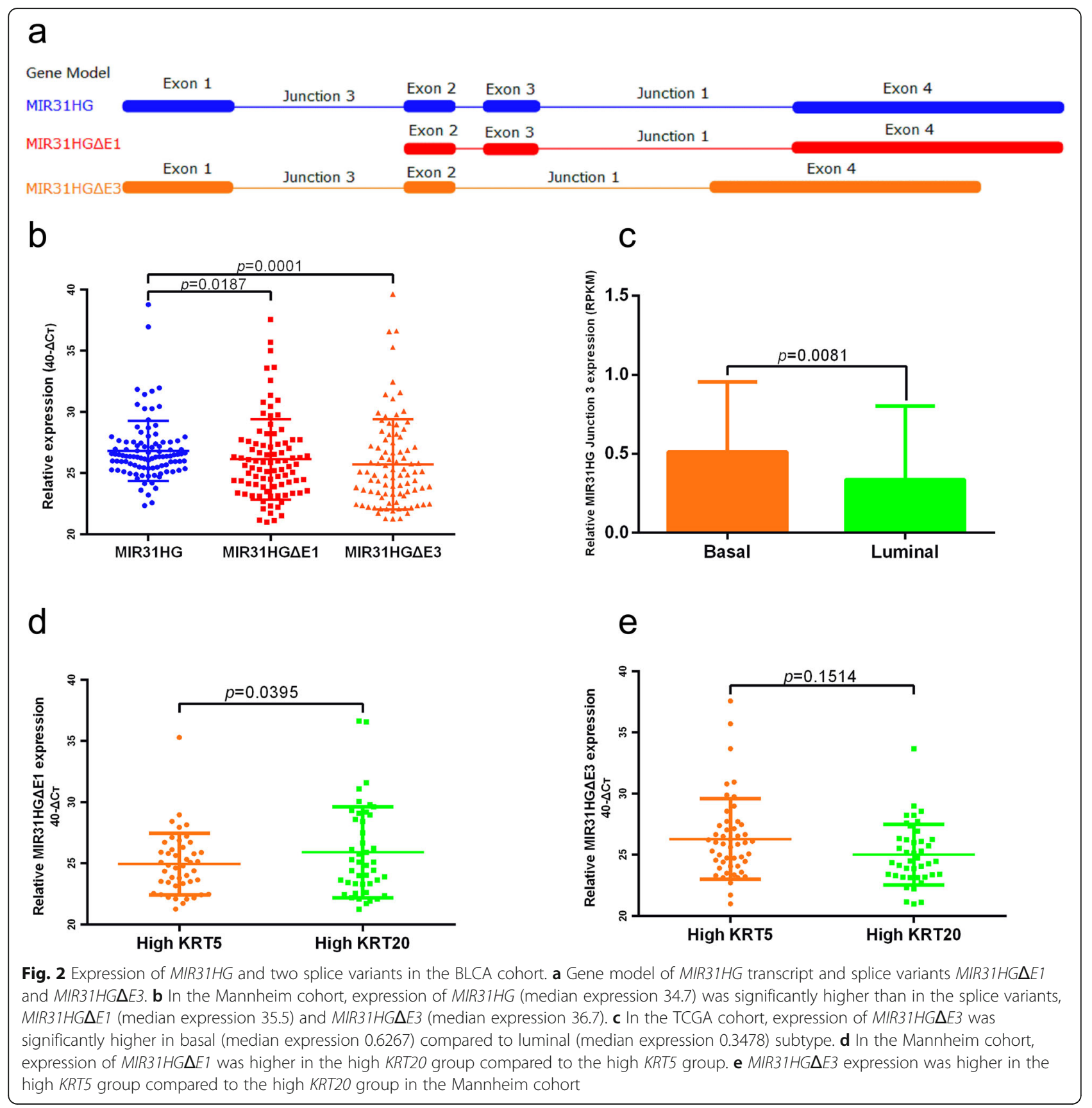

cohort (Fig. S2). Interestingly, survival analysis showed that patients of the TCGA cohort with basal subtype were significantly associated with OS and DFS based on MIR31HG risk stratification. The group with high MIR31HG expression showed a worse OS compared to the group with low expression (median survival, 28 vs. 16 months, $p=0.0073$, Fig. 4a). Furthermore, the group with high MIR31HG expression showed a worse DFS compared to the group with low expression (median survival, 27 vs. 15 months, $p=0.1379$, Fig. 4b).

\section{Splice variants of MIR31HG as prognostic markers for patients}

In the Mannheim cohort, Kaplan-Meier analysis and the log-rank test were used to evaluate the association of the expression of two MIR31HG splice variants with $O S$ and DFS. For the full-length transcript of MIR31HG, no significant difference in OS (median survival 18 vs. 21 months, $p=0.2032$, Fig. 5a) and DFS (median survival 11 vs. 9 months for MIR31HG, $p=0.7365$, Fig. 5b) was found between high and low 


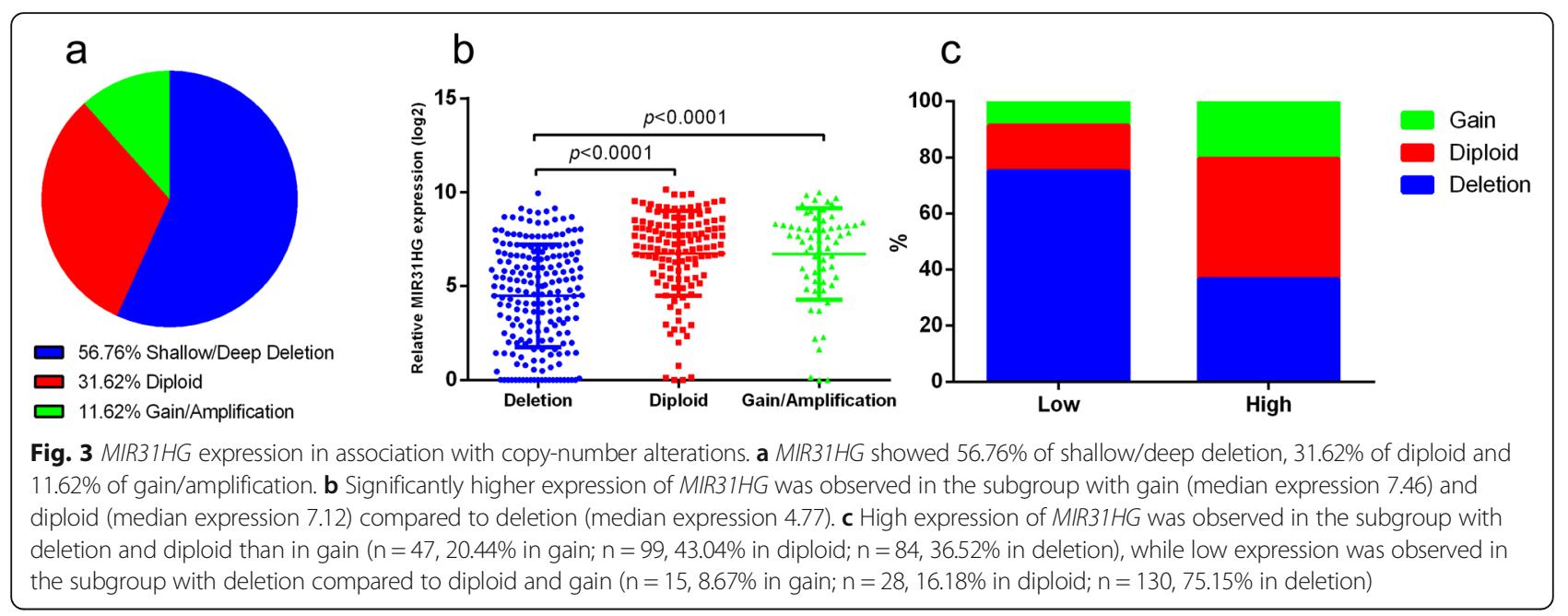

expression levels. Tumors with both high MIR31HGAE1 (median survival 15 vs. 38 months, $p=$ 0.0315 , Fig. 5c) and MIR31HGAE3 expression (median survival 12 vs. 30 months, $p=0.0024$, Fig. 5e) showed a worse OS. The groups with high MIR31HGAE1 (median survival 7 vs. 25 months, $p=0.0134$, Fig. 5d) and MIR31HGAE3 (median survival 9 vs. 15 months, $p=0.1008$, Fig. 5f) expression also showed a worse DFS. Tumors with high expression of MIR31HG Exon1-2 (Junction 3), which could partially present as MIR31HGAE3, showed a worse OS than the group with low expression, in the TCGA cohort with basal subtype (median survival 15 vs. 17 months, $p=0.0298$, Fig. S3A). No significant difference was found for DFS (median survival 12 vs. 15 months, $p=0.5670$, Fig. S3B).
Similar to the analysis of MIR31HG in the TCGA cohort, the expression of MIR31HG and its splice variants were also analyzed regarding to OS and DFS in patients from Mannheim with basal subtype (high KRT5 expression). No significant differences were found in OS (median survival 18 vs. 20 months, $p=0.6664$, Fig. S4A) and DFS (median survival 17 vs. 15 months, $p=0.8690$, Fig. S4B) between high and low expression of MIR31HG, also in high and low expression of MIR31HGAE1 (median survival 15 vs. 18 months for OS, $p=0.2338$, Fig. S4C; median survival 29 vs. 17 months for DFS, $p=0.5984$, Fig. S4D). Significant difference with OS was found between high and low expression of MIR31HGAE3 in the basal subtype cohort (median survival 13 vs. 22 months, $p=0.0194$, Fig. S4E). Yet, no significant correlation was found with DFS (median survival 15 vs. 36 months, $p=0.3427$, Fig. S4F).

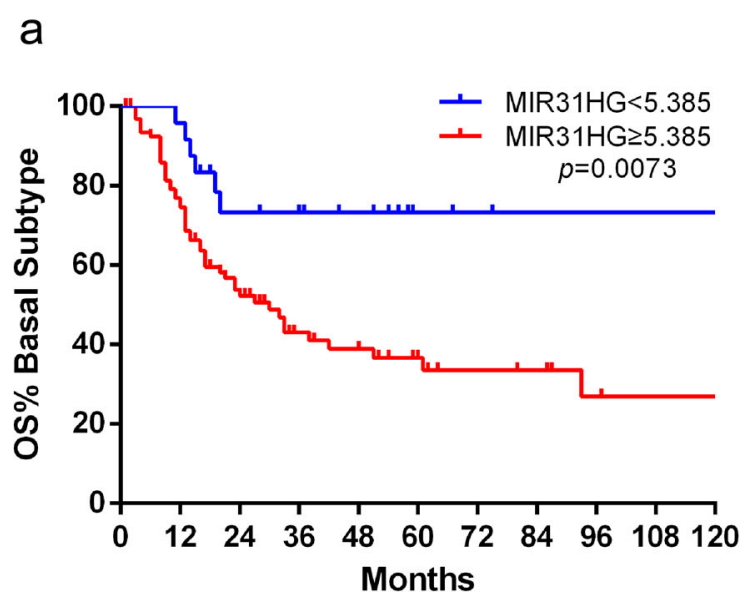

b

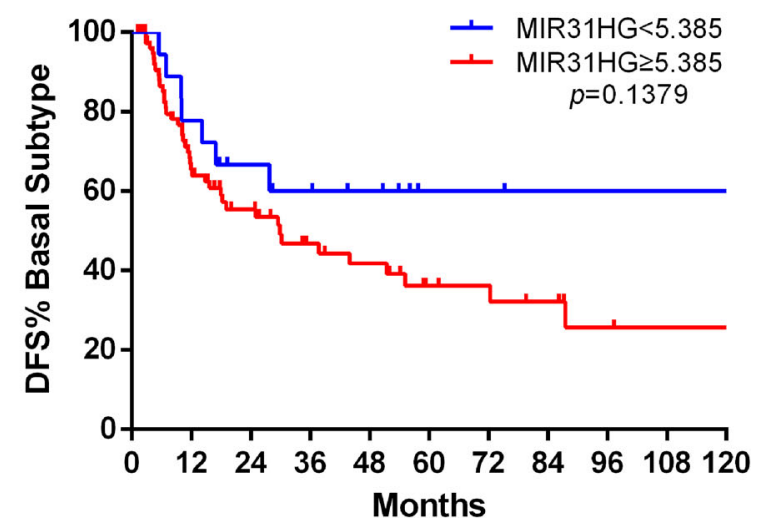

Fig. 4 Kaplan-Meier plot of overall survival (OS) and disease free survival (DFS) were associated with MIR31HG risk stratification. a In the TCGA cohort with basal subtype, the group with high MIR31HG expression showed a worse OS compared to the group with low expression (median survival, 28 vs. 16 months, $p=0.0073$ ). $\mathbf{b}$ In the TCGA cohort with basal subtype, the group with high MIR31HG expression showed a worse DFS compared to the group with low expression (median survival, 27 vs. 15 months, $p=0.1379$ ). The numbers below the figures showed the number of patients at risk in each group 

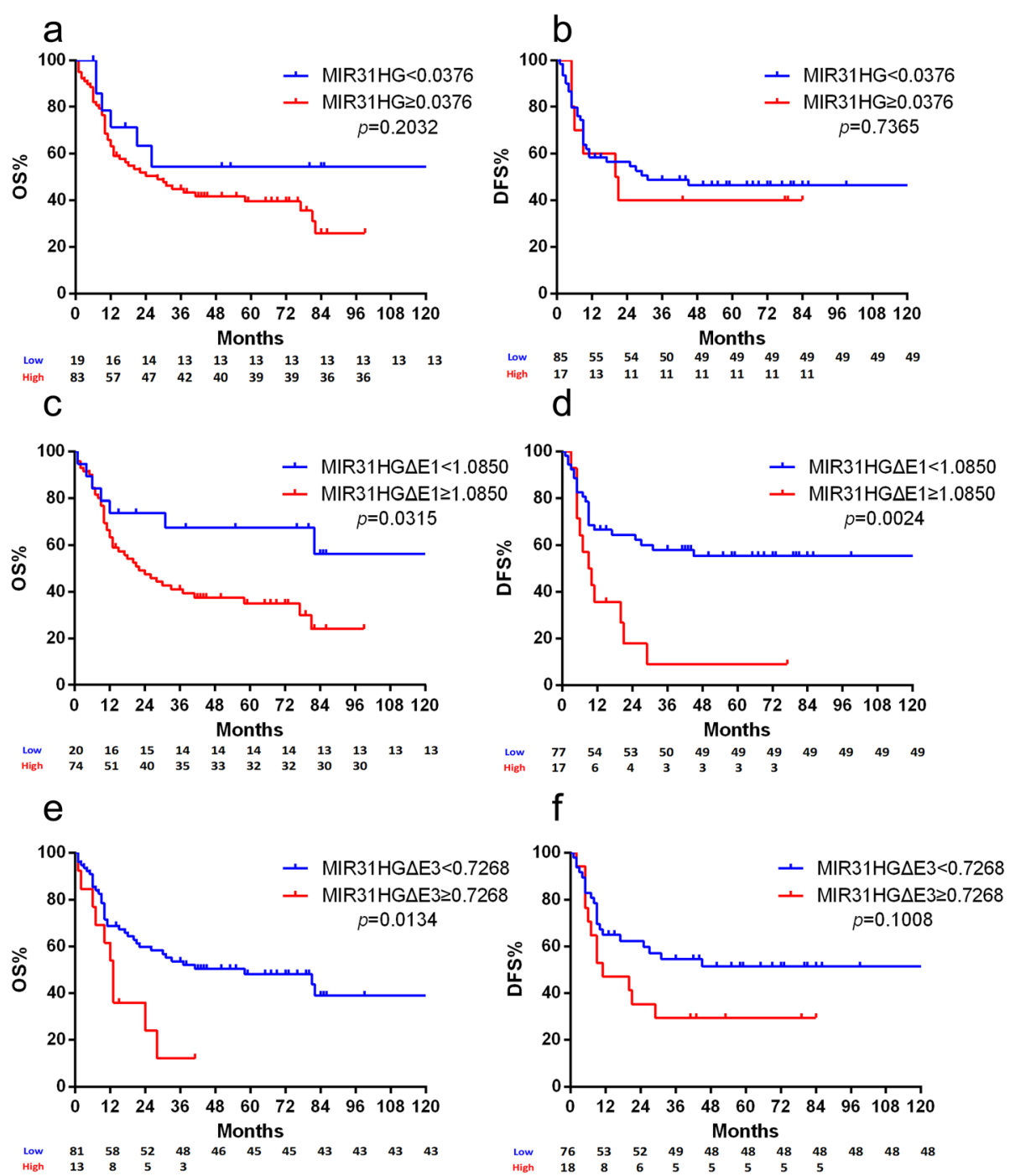

Fig. 5 Kaplan-Meier plot of the Mannheim cohort of OS and DFS associated with MIR31HG and its splice variants risk stratification. No significant correlation was found with OS (a, median survival 18 vs. 21 months) and DFS (b, median survival 11 vs. 9 months for MIR31HG) in the group with full-length transcript of MIR31HG. The group with high MIR31HGDE1 expression showed a worse OS (c, median survival 15 vs. 38 months) and DFS (d, median survival 7 vs. 25 months) compared to the group with low expression. The group with high MIR31HG $\Delta$ E3 expression showed a worse OS (e, median survival 12 vs. 30 months) and DFS (F, median survival 9 vs. 15 months) compared to the group with low expression. The numbers below the figures showed the number of patients at risk in each group

In the univariable Cox regression analysis, MIR31HGAE1 $(p=0.0063)$ and MIR31HGLE3 $(p=0.0114)$ expression as well as the stage of cancer $(p=0.0092)$ were found to be predictive for patient outcome. In the multivariable Cox regression analysis, MIR31HGAE3 was identified as an independent prognostic factor $(p=0.0090)$, similar as stage $(p=$ 0.0243). No significant differences of OS were found between basal (high KRT5) and non-basal (low KRT5) group in the univariable analysis. However, no significant correlation was observed between the full-length transcript of MIR31HG regarding to OS of the patients in the univariable analysis, nor with patient age, patient gender, lymph node status, and LVI (Table 2).

\section{MIR31HG is required for BLCA tumorigenesis}

To evaluate the possible role of MIR31HG in BLCA, a pool of four siRNAs against MIR31HG (designated siMIR31HG, set of 4) were transfected in T24, UMUC3, and SCaBER cells. Each transfection resulted in a sufficient knocked down of MIR31HG expression. Cell viability was determined using the MTS assay based on absorbance at $490 \mathrm{~nm}$ after $0,24,48$, and $72 \mathrm{~h}$. T24, UMUC3, and SCaBER cells with MIR31HG knockdown by siRNA showed lower cellular viability at three time points compared to the control group transfected with scramble siRNA (si-NC, Fig. 6a-c). Moreover, colony formation was detected by culturing cells in a 6-well 
Table 2 Univariable and multivariable cox regression analysis of MIR31HG and its splice variants with clinicopathological features in the Mannheim cohort (HR hazard ratio, Cl confidence interval, LVI lymphovascular invasion, Basal high KRT5 expression, Non-basal low KRT5 expression, significant $p$ values are bold)

\begin{tabular}{|c|c|c|c|c|}
\hline \multirow[t]{2}{*}{ Factor } & \multicolumn{2}{|l|}{ Univariable } & \multicolumn{2}{|l|}{ Multivariable } \\
\hline & HR (95\% Cl) & $p$ & $\mathrm{HR}(95 \% \mathrm{Cl})$ & $p$ \\
\hline \multicolumn{5}{|l|}{ Diagnosis Age } \\
\hline$<70$ vs. $\geq 70$ & $0.701(0.406-1.212)$ & 0.2041 & - & - \\
\hline \multicolumn{5}{|l|}{ Gender } \\
\hline Male vs. Female & 0.980(0.535-1.795) & 0.9485 & - & - \\
\hline \multicolumn{5}{|l|}{ Stage } \\
\hline T2 vs. T3/4 & $0.401(0.202-0.797)$ & 0.0092 & $0.445(0.220-0.900)$ & 0.0243 \\
\hline \multicolumn{5}{|l|}{ LVI } \\
\hline Negative vs. Positive & $0.550(0.075-4.050)$ & 0.5570 & - & - \\
\hline \multicolumn{5}{|l|}{ Lymphnode Status } \\
\hline Negative vs. Positive & $0.547(0.245-1.221)$ & 0.1418 & $0.694(0.243-1.987)$ & 0.4961 \\
\hline \multicolumn{5}{|l|}{ Molecular Subtype } \\
\hline Basal vs. Non-basal & $1.202(0.596-2.422)$ & 0.6073 & - & - \\
\hline \multicolumn{5}{|l|}{ MIR31HG } \\
\hline Low vs. High & $1.720(0.681-4.347)$ & 0.2522 & - & - \\
\hline \multicolumn{5}{|l|}{ MIR31HG $\triangle E 1$} \\
\hline Low vs. High & $3.753(1.457-9.664)$ & 0.0063 & $1.896(0.831-4.329)$ & 0.1294 \\
\hline \multicolumn{5}{|l|}{ MIR31HGDE3 } \\
\hline Low vs. High & $2.522(1.235-5.149)$ & 0.0114 & $2.805(1.293-6.083)$ & 0.0090 \\
\hline
\end{tabular}

plate and stained with crystal violet after 7 days. T24, UMUC3, and SCaBER cells with MIR31HG knockdown showed fewer cell colonies compared to the control group (Fig. 6d). The staining intensity of the colony formation assay was quantified by calculating the colony intensity percentage. BLCA cells with MIR31HG knockdown showed a reduced formation of colonies compared to the control group (Fig. 6e). Migration was detected by a wound-healing assay. T24, UMUC3, and SCaBER cells with MIR31HG knockdown showed a larger open wound area compared with the control group after $12 \mathrm{~h}$ (Fig. 6f). The difference in open wound area after $12 \mathrm{~h}$ was quantified by calculating the percentage of change in the open wound area (open wound area at 12 $\mathrm{h}$ - open wound area at $0 \mathrm{~h}$ ). BLCA cells with MIR31HG knockdown showed fewer changes in open wound area compared with the control group (Fig. 6g).

\section{Two splice variants of MIR31HG regulate BLCA growth with cell specificity}

To further evaluate the possible role of the transcripts MIR31HGAE1 and MIR31HG E3 in BLCA, transcriptspecific siRNAs were transfected into BLCA cells. Cell viability was quantified by MTS assay based on absorbance at $490 \mathrm{~nm}$ after 0, 24, 48, and $72 \mathrm{~h}$. T24, UMUC3, and SCaBER cells with MIR31HGAE1 and MIR31HGAE3 knockdown by specific siRNA showed decreased cellular viability with cell specificity (Fig. 7a-c). MIR31HG $\triangle E 1$ knockdown showed significantly decreased absorbance in T24 ( $p=0.0018$, Fig. 7a) and UMUC3 ( $p=0.0029$, Fig. 7b) cells after $72 \mathrm{~h}$, and MIR31HG $\triangle E 3$ knockdown resulted in a significant decrease in absorbance in SCaBER $(p=$ 0.0346 , Fig. 7c) cells exclusively after $72 \mathrm{~h}$. Colony formation was detected by culturing cells in 6-well plates and staining with crystal violet after 7 days. T24, UMUC3, and

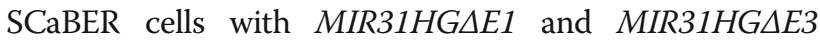
knockdown by specific siRNA showed fewer cell colonies with cell specificity (Fig. 7d). T24 and UMUC3 cells with MIR31HGAE1 knockdown showed a significantly reduced staining intensity compared with control transfected cells. SCaBER cells with MIR31HG significantly decreased staining intensity (Fig. 7e). Migration was detected with a wound healing assay using cell culture inserts. T24, UMUC3, and SCaBER cells with MIR31HGDE1 and MIR31HGDE3 knockdown by specific siRNA showed a larger open wound area compared with cell specificity after $12 \mathrm{~h}$ (Fig. 7f). The difference in open wound area after $12 \mathrm{~h}$ was quantified by calculating the percentage of change in open wound area (open wound area $12 \mathrm{~h}$ - open wound area $0 \mathrm{~h}$ ). T24 and UMUC3 cells showed fewer changes in open wound area, both in the MIR31HGAE1 and MIR31HG $\triangle E 3$ knockdown groups. SCaBER cells showed fewer changes in the open wound area upon MIR31HGAE3 knockdown (Fig. 7g). 


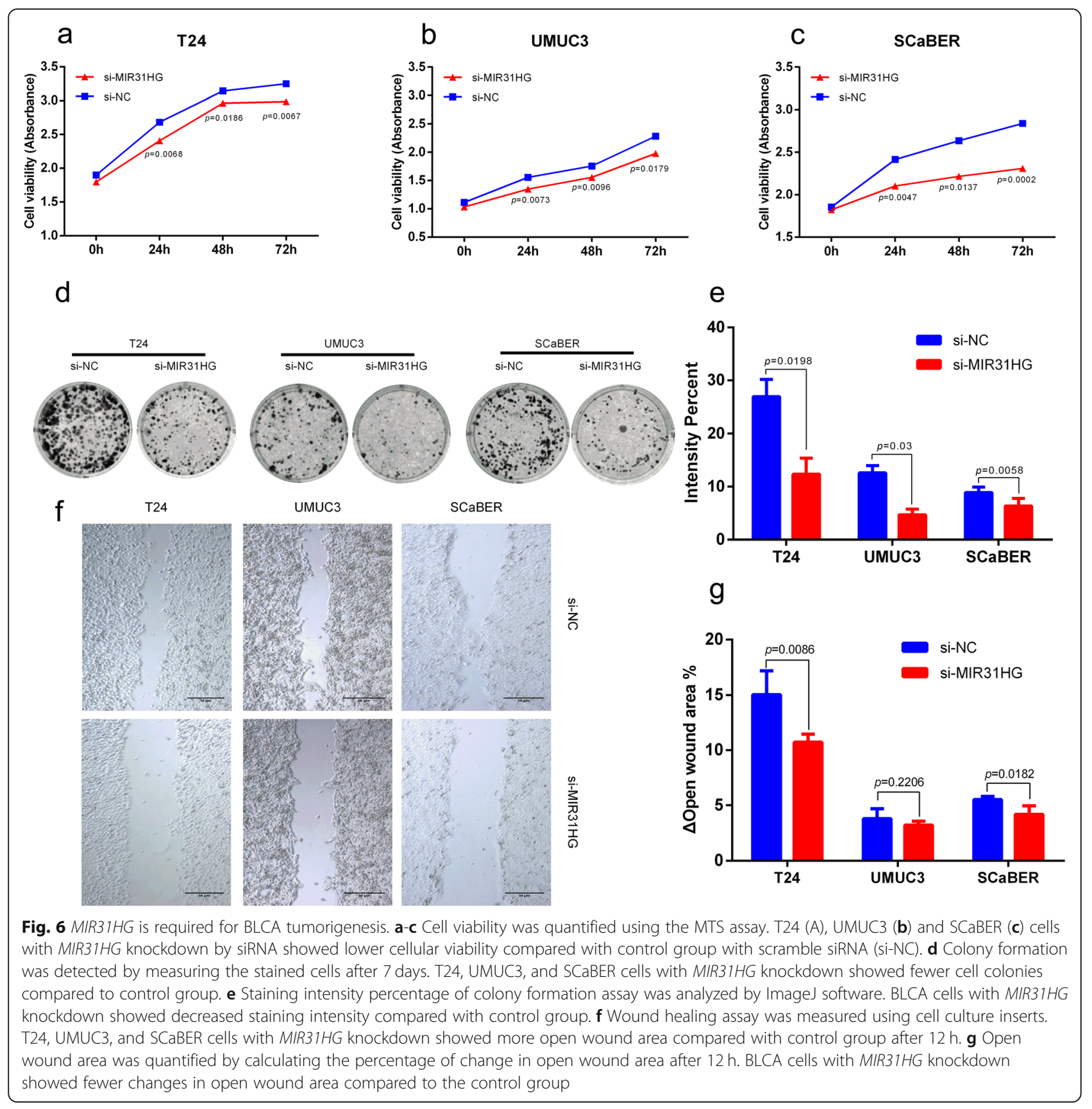

\section{MIR31HG is associated with EGFR pathway}

To discover the potential mechanisms underlying the functions of MIR31HG, the related proteins were focused on EGFR, which was reported as a molecular signature of basal or squamous-like bladder cancer [34]. The lncRNA-protein interactions of MIR31HG and EGFR were analyzed by lncPro [24] and predicted 9 Isoforms of EGFR interacting. Besides EGFR and its isoforms, MIR31HG was predicted as interactive with phosphoinositide 3-kinase (PI3K) and receptor tyrosineprotein kinase erbB-2 (HER2) protein (score above 50, Table 3).
To further reveal the interaction with the coded gene, gene expression of EGFR was detected in MIR31HG knockdown cells. Significantly higher expression of EGFR was observed in SCaBER cells with MIR31HG knockdown compared to the control group ( $p=0.0367$, Fig. 8). No significant difference of expression was observed between T24 $(p=0.4086)$ and UMUC3 $(p=0.4734)$ cells with MIR31HG knockdown and control group (Fig. 8).

\section{Discussion}

This study aimed to investigate the expression pattern and biological function of MIR31HG in MIBC, as well as its 


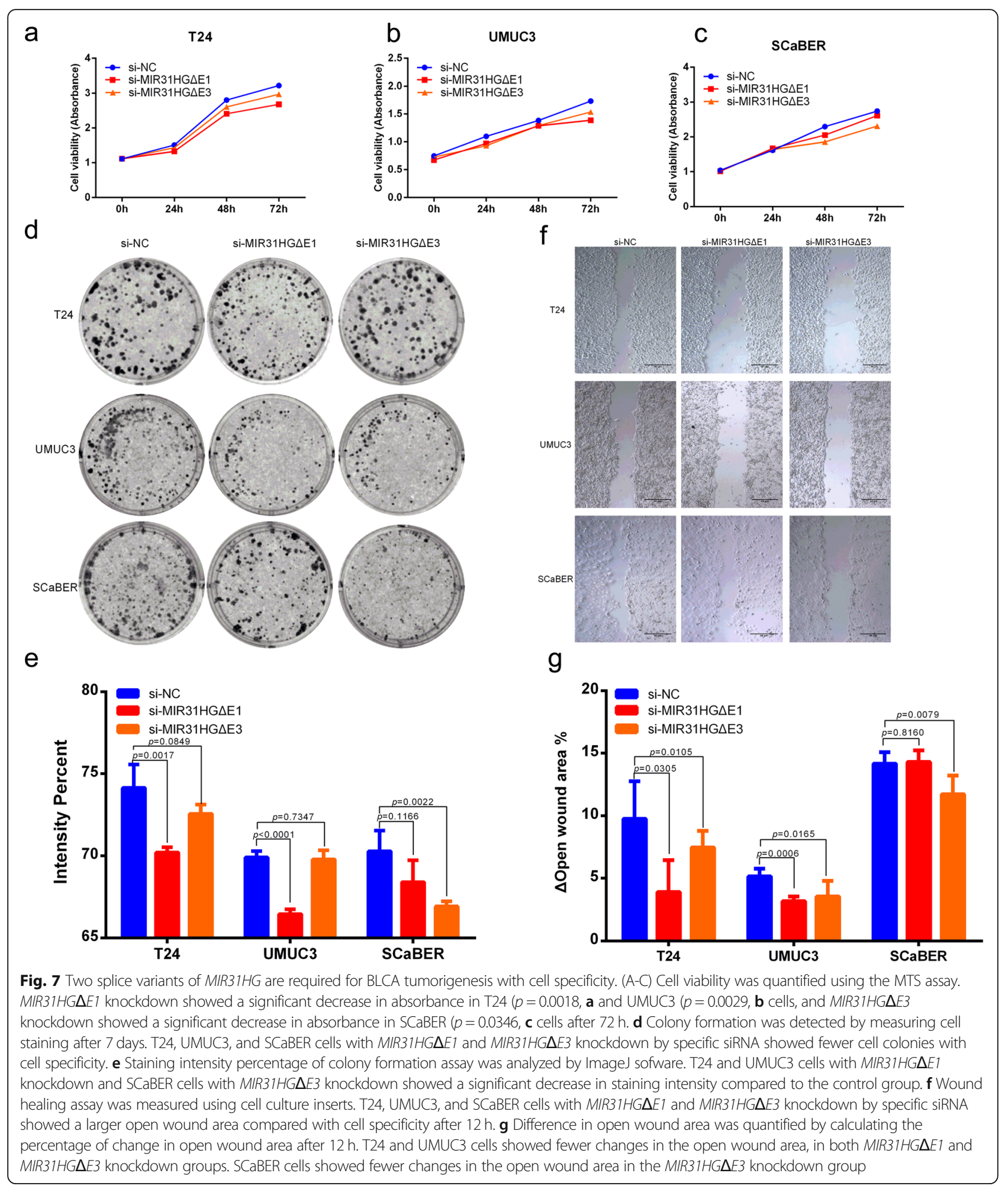

clinical significance and prognostic value in patients. In order to evaluate its role in tumorigenesis and progression, functional in vitro assays were combined with lncRNA expression analysis based on molecular subtypes and relevant clinicopathologic parameters.
In previous studies on multifarious tumors, MIR31HG showed a tissue-specific expression pattern. In breast cancer and non-small cell lung cancer (NSCLC) cells, MIR31HG expression was upregulated [11, 35]. In gastric cancer tissues and cell lines, MIR31HG was poorly 
Table 3 Interaction scores of EGFR, PI3K HER2 protein and MIR31HG

\begin{tabular}{ll}
\hline Protein & Score \\
\hline EGFR & 72.4935 \\
EGFR isoform a & 75.0794 \\
EGFR isoform b & 93.4501 \\
EGFR isoform c & 83.9972 \\
EGFR isoform d & 93.8757 \\
EGFR isoform e & 72.5006 \\
EGFR isoform f & 75.7342 \\
EGFR isoform g & 73.3893 \\
EGFR isoform h & 82.7141 \\
EGFR isoform i & 84.5429 \\
PI3K & 75.5458 \\
HER2 isoform a & 70.9194 \\
HER2 isoform b & 82.7639 \\
HER2 isoform c & 79.0902 \\
HER2 isoform d & 69.6083 \\
HER2 isoform e & 91.4699 \\
\hline
\end{tabular}

expressed [13]. Another study showed that MIR31HG level is substantially upregulated in oral carcinoma, significantly associated with poor clinical outcomes and representing an independent prognostic predictor [36]. In our study, lower MIR31HG transcript levels were found in luminal-like and mixed-type BLCA cell lines compared with a normal urothelium cell line. Accordingly, down-regulated MIR31HG expression was found in cancer tissues compared to normal tissues, which supports expression results measured by qPCR from a previous study [15]. However, the previous results were measured in stage- and type-mixed BLCA tissues, and in this study, MIR31HG was measured in MIBC and associated with multiple molecular subtype respectively. In contrast, MIR31HG was found to be highly expressed in cells lines and clinical tumor samples with the basal subtype compared to luminal and other subtypes, indicating that MIR31HG not only shows tissue specific, but also subtype-specific overexpression in MIBC.

In contrast to a previous study, which reported that miR-31 and MIR31HG are down-regulated in triplenegative breast cancer (TNBC) cell lines of basal subtype [37], the present study shows that MIR31HG is highly expressed in the BLCA cell line of basal subtype and markedly correlates with the survival of patients with MIBC basal subtype. This might be due to tissue specific expression of MIR31HG. In this study, two MIBC cohorts with multiple molecular subtypes were involved rather than single cell line, which may also lead to the dissimilar results. Further studies of BLCA preclinical models are needed for validation. It is noteworthy that two transcript variants of MIR31HG (MIR31HG $E 1$ and MIR31HGAE3) were identified and their expression was analyzed in BLCA cells and MIBC tissues. Besides the different expression levels in MIBC patient tissues, the two splice variants showed distinguished expression patterns in basal and luminal subtypes, respectively. MIR31HGAE3 showed high expression in the basal subtype, both in the TCGA cohort and the Mannheim cohort, which is also observed for the group with high KRT5 expression. In contrast, MIR31HGDE1 showed

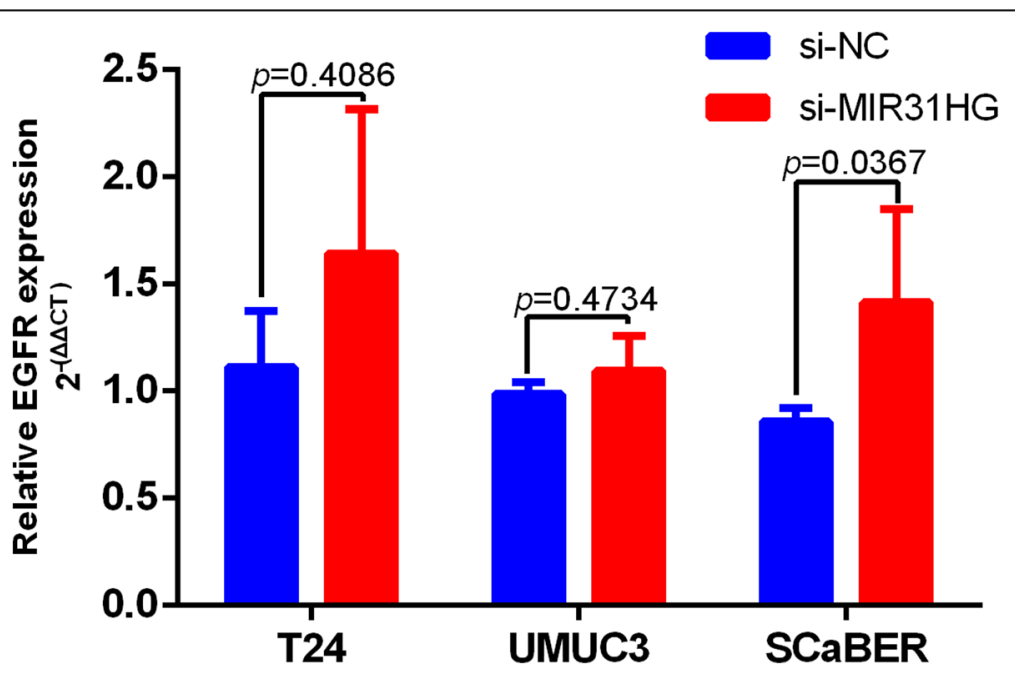

Fig. 8 Expression of EGFR in BLCA cell lines with MIR31HG knockdown. To narrow the differences in EGFR expression between cell lines, the $2^{-\triangle \Delta C T}$ values of expression data in T24, UMUC3 and SCaBER cells were normalized by the expression data in UROtsa. EGFR expression was significantly higher in SCaBER cells with MIR31HG knockdown compared to negative siRNA group. Expression of EGFR showed no significant difference between T24 and UMUC3 cells with MIR31HG knockdown and negative siRNA group 
high expression in luminal subtype tumors in the Mannheim cohort, corresponding to tumors with a high KRT20 expression.

Additionally, higher MIR31HG transcript levels were found to be associated with worse OS and DFS in the basal subtype cohort, but not in the whole TCGA cohort. It is the first time to discover the prognostic value of MIR31HG in BLCA, or associated with subtypes of tumors. Furthermore, expression of MIR31HGDE1 and MIR31HGDE3 was significantly associated with OS and DFS in the Mannheim cohort, rather than the full-length transcript of MIR31HG. In the TCGA cohort, it was demonstrated that MIR31HGAE3 expression was significantly associated with OS of the basal subtype group. These results, together with univariable and multivariable Cox regression analysis suggested that the alternative splice variants of MIR31HG may serve as potential biomarkers for certain molecular subtypes of MIBC, which could contribute to an individualized bladder cancer subclassification and therapy decision making. It is worth noting that lymph node status is not associated with OS based on survival analysis, which is controversial to other studies [38]. The possible reasons could be limited number of patients, diverse distribution of lymph node status and $\mathrm{T}$ stages as well as different scale of lymph node examination. Therefore, validation in larger patient cohorts with long-term follow-up is needed. In the Mannheim cohort, few patients were treated with neoadjuvant or adjuvant chemotherapy. For further validation, the number of positive lymph nodes and chemotherapy status should be also taken into consideration.

Two groups independently recognized the significance of a distinct basal MIBC subtype [39, 40]. According to classification of the TCGA, basal tumors were divided into two subsets that were largely distinguished by differential expression of biomarkers associated with EMT, which is a reversible developmental process by promoting invasion, metastasis, "stemness", and drug resistance [32, 41]. The potential significance of the mesenchymal basal BLCA was identified using a "claudin-low" gene expression signature in breast cancer [42]. A previous study reported that tumor suppressor microRNA-361 was de-repressed by MIR31HG in osteosarcoma cells, leading to cell growth and mesenchymal phenotype [43]. These results may indicate that high expression MIR31HG could be served as a surrogate marker of poor outcome defined by relative activation of EMT and sponge of tumor suppressor. The discovery that MIR31HG is highly expressed and significantly outcomecorrelated in MIBC with basal subtype, has complemented the selection of MIBC markers.

Due to its potential clinical relevance as a marker, the expression of MIR31HG and its underlying biological functions could be vital to the tumor. In this study, by knocking down MIR31HG expression using siRNA, diminished cell proliferation, colony formation, and migration were assessed in BLCA cell lines. This study is the first to highlight the function of MIR31HG in BLCA cells, which indicates that MIR31HG might serve as an oncogene in certain types of BLCA. With the in-depth study of MIR31HG, several downstream targets were found in present researches. For example, it is reported that overexpression of MIR31HG significantly decreased the expression of miR-575, enhanced the suppression of tumorigenicity 7 like (ST7L) in hepatocellular carcinoma (HCC). Thus, MIR31HG regulated ST7L expression through sponging miR-575, and acted as tumor suppressor in HCC [44]. Another published research showed that the level of MIR31HG in esophageal squamous cell carcinoma (ESCC) tissues was positively correlated with the expression of furin and matrix metalloproteinase 1 (MMP1). When MIR31HG was silenced, the expressions of furin and MMP1 in ESCC cells were significantly inhibited. These results suggest that the involvement of MIR31HG in invasion and migration of ESCC cell may be partly achieved through the furin / MMP1 pathway [45]. In a study in oral cancer, MIR31HG was identified as a hypoxia-inducible lncRNA and forms a complex with hypoxia-inducible factor-1 $\alpha$ (HIF-1 $\alpha)$, thus as an adverse prognostic predictor for the cancer progression [36]. In addition, it was reported that MIR31HG could function as an oncogene that promotes pancreatic cancer progression, by acting as an endogenous sponge competing for miR-193b [14]. Similarly, it was shown that silencing of MIR31HG significantly inhibited NSCL $\mathrm{C}$ cell migration, invasion, and metastasis by attenuated sponging of miR-214 [35]. The various mechanisms of MIR31HG suggest that downstream pathways could be involved with other non-coding RNAs, which requires further verification. In this study, similar to the fulllength transcript, a series of functional experiments validated the corresponding roles in certain types of BLCA cells. The knock-down of MIR31HGDE3 resulted in reduced colony formation ability and cell viability solely in SCaBER cells, suggesting that exon-specific or transcript-specific mechanisms could be a new direction in studying basal-like BLCA. Recently, emerging data have demonstrated that RNA splice variants are associated with drug resistance in cancer. The expression of androgen receptor (AR) splicing variants in castrationresistant prostate cancer (CRPC) samples increased significantly. The most common AR splicing variants are AR-V7 and ARv567es [46, 47]. These variants are important factors for insensitive to AR antagonists of CRPC patients. Furthermore, splice variants in V600E BRAF-mutant-positive malignant melanoma patients were shown to be associated with vemurafenib resistance, indicating that aberrant splicing could be a novel 
mechanism of acquired resistance [48]. Furthermore, interference of the pre-mRNA splicing modulators sufficiently inhibited formation of these splice variants, suggesting that splice variant-specific siRNAs may be proposed as a therapeutic strategy to overcome drug resistance [49].

For basal or squamous-like bladder cancer, molecular signatures were found based on clustering RNA-seq data, including EGFR [34]. EGFR is overexpressed in up to $74 \%$ of bladder cancer tissue specimens, and is amplified in squamous cell carcinomas (SCC) of the bladder [50]. To further investigate the potential relationship between MIR31HG and EGFR, a computational method called lncPro was applied to predict the associations within. All the nine isoforms of EGFR, as well as PI3K and HER2 protein, were predicted as interactive with MIR31HG. The positive interaction score suggested that MIR31HG might be involved in the EGFR/PI3K/AKT signaling pathway. Furthermore, expression of EGFR was detected in three MIR31HG-knockdown BLCA cell lines. In SCaBER cells, which shows the basal / squamous signature, expression of EGFR was reversely correlated with MIR31HG. According to another study on lung cancer, by knocking down MIR31HG, reversal of gefitinib resistance was found by regulation of the EGFR/PI3K/AKT signaling pathway [51]. Taken together, these findings suggested that MIR31HG might potentially correlate with the EGFR pathway.

\section{Conclusions}

In summary, our data show that MIR31HG was highly expressed in basal subtype cells and tissues of MIBC. MIR31HG and its splice variants (MIR31HGAE1 and MIR31HGAE3) could regulate proliferation and migration of corresponding BLCA cells. Furthermore, in different MIBC cohorts, MIR31HG and its splice variants were associated with survival of different subgroups of patients. Our research provides new insights into studies for the molecular classification of MIBC.

\section{Supplementary Information}

The online version contains supplementary material available at https://doi. org/10.1186/s13046-020-01795-5

\footnotetext{
Additional file 1: Fig. S1. Expression of MIR31HG in BLCA tissue samples and cell lines based on in silico data. (A) Expression of MIR31HG was higher in basal (median expression 7.10 with range of 0 to 9.96) subtype than in luminal (median expression 5.21 with range of 0 to 10.16) and infiltrated (median expression 4.99 with range of 0 to 9.16) subtypes in patients of the TCGA cohort. (B) No significant difference of MIR31HG expression was found between lymph node metastasis negative and positive groups. (C) RNA-seq data from the Cancer Cell Line Encyclopedia showed expression levels in TPM (transcripts per million) for MIR37HG in 25 BLCA cell lines. Fig. S2. Kaplan-Meier plot of the TCGA cohort of OS and DFS associated with MIR31HG risk stratification. The group with high or low expression of MIR31HG showed no significant correlation with OS (A, median survival, 16 vs. 15 months, $p=0.9638$ ) and DFS
}

( $\mathrm{B}$, median survival, 15 vs. 17 months, $p=0.4175$ ) in the whole TCGA cohort. The numbers below the figures showed the number of patients at risk in each group. Fig. S3. Kaplan-Meier plot of the TCGA cohort with basal subtype of overall survival associated with MIR31HG Exon1-2 (Junction 3) risk stratification. (A) The group with high Junction 3 expression showed worse OS than the group with low expression (median survival, 17 vs. 14 months, $p=0.0298$ ). (B) No significant difference was observed in DFS between the group with high and low Junction 3 expression (median survival, 17 vs. 15 months, $p=0.5670$ ). The numbers below the figures showed the number of patients at risk in each group. Fig. S4. Kaplan-Meier plot of the Mannheim cohort with basal subtype of OS and DFS associated with MIR31HG and its splice variants risk stratification. No significant correlation was found with OS (A, median survival 18 vs. 20 months) and DFS (B, median survival 17 vs. 15 months) in the group with full-length transcript of MIR31HG. No significant correlation was found with OS ( $C$, median survival 15 vs. 18 months) and DFS ( $D$, median survival 29 vs. 17 months) in the group with MIR31HGDE1. The group with high MIR31HG $\triangle E 3$ expression showed a worse OS ( $E$, median survival 13 vs. 22 months) compared to the group with low expression, no significant correlation was found with DFS ( $F$, median survival 15 vs. 36 months). The numbers below the figures showed the number of patients at risk in each group. Table S1. siRNAs used in this study. Table S2. Primers and probes used in this study.

\section{Abbreviations}

BLCA: Bladder urothelial carcinoma; DFS: Disease-free survival; EGFR: Epidermal growth factor receptor; FFPE: Formalin fixed paraffin embedded; IncRNA: Long non-coding RNA; MIR31HG: miR-31 host gene; MIBC: Muscle invasive bladder cancer; OS: Overall survival; siRNA: small interfering RNA; TCGA: The Cancer Genome Atlas; TPM: Transcripts per million

\section{Acknowledgments}

The authors would like to thank Annette Steidler and Lena Hoffmann for excellent technical support. We would like to thank Blerta Thaqi for the technical work.

\section{Authors' contributions}

SW: Conceptualization, Methodology, Investigation, Writing - original draft. KN: Methodology, Investigation, Writing - review\& editing. TSW: Investigation, Writing - review\& editing. AF: Formal analysis, Investigation. C-AW: Writing review \& editing. ME: Writing - review \& editing. SP: Writing - review \& editing. MK: Writing - review \& editing. PE: Conceptualization, Methodology, Funding acquisition, Supervision, Writing - review \& editing. The author(s) read and approved the final manuscript.

\section{Funding}

The study was funded by the common scientific pool of participating institutions. Sheng Wu was supported by a scholarship from the China Scholarship Council. Open Access funding enabled and organized by Projekt DEAL.

Availability of data and materials

The data and materials of this study are available from the corresponding author for reasonable requests.

\section{Ethics approval and consent to participate}

Studies involving human participants were approved by the ethical board of the University of Heidelberg (2015-549 N-MA) and performed in accordance with relevant guidelines and regulations.

\section{Consent for publication}

All authors agree to submit the article for publication.

\section{Competing interests}

The authors declare that they have no conflict of interest.

\section{Author details}

${ }^{1}$ Department of Urology and Urosurgery, Medical Faculty Mannheim, University of Heidelberg, 68167 Mannheim, Germany. 'Department of 
Oncology, The First Affiliated Hospital of Anhui Medical University, Hefei 230032, Anhui, China. ${ }^{3}$ Institute of Pathology, Medical Faculty Mannheim, University of Heidelberg, 68167 Mannheim, Germany. ${ }^{4}$ Institute of Pathology, University Hospital Erlangen, Friedrich-Alexander-University

Erlangen-Nürnberg, 91052 Erlangen, Germany.

Received: 25 May 2020 Accepted: 2 December 2020

Published online: 17 December 2020

\section{References}

1. Bray F, Ferlay J, Soerjomataram I, Siegel RL, Torre LA, Jemal A. Global cancer statistics 2018: GLOBOCAN estimates of incidence and mortality worldwide for 36 cancers in 185 countries. CA Cancer J Clin. 2018;68(6):394-424.

2. Babjuk M, Bohle A, Burger M, Capoun O, Cohen D, Comperat EM, et al. EAU guidelines on non-muscle-invasive Urothelial carcinoma of the bladder: update 2016. Eur Urol. 2017;71(3):447-61.

3. Aragon-Ching JB, Werntz RP, Zietman AL, Steinberg GD. Multidisciplinary Management of Muscle-Invasive Bladder Cancer: current challenges and future directions. Am Soc Clin Oncol Educ Book. 2018;38:307-18.

4. Choi W, Porten S, Kim S, Willis D, Plimack ER, Hoffman-Censits J, et al. Identification of distinct basal and luminal subtypes of muscle-invasive bladder cancer with different sensitivities to frontline chemotherapy. Cancer Cell. 2014;25(2):152-65

5. Li Y, Yang K, Li K, Liu H, Zhao S, Jiao M, et al. Clinical and molecular characteristics of bladder urothelial carcinoma subtypes. J Cell Biochem. 2019:120(6):9956-63.

6. Volkmer J-P, Sahoo D, Chin RK, Ho PL, Tang C, Kurtova AV, et al. Three differentiation states risk-stratify bladder cancer into distinct subtypes. Proc Natl Acad Sci. 2012;109(6):2078-83.

7. Wang KC, Chang HY. Molecular mechanisms of long noncoding RNAs. Mol Cell. 2011;43(6):904-14.

8. Gibb EA, Brown CJ, Lam WL. The functional role of long non-coding RNA in human carcinomas. Mol Cancer. 2011;10(1):38.

9. Chen J, Miao Z, Xue B, Shan Y, Weng G, Shen B. Long non-coding RNAs in urologic malignancies: functional roles and clinical translation. J Cancer. 2016;7(13):1842-55.

10. Prensner JR, Chinnaiyan AM. The emergence of IncRNAs in cancer biology. Cancer Discov. 2011;1(5):391-407

11. Shi Y, Lu J, Zhou J, Tan X, He Y, Ding J, et al. Long non-coding RNA Loc554202 regulates proliferation and migration in breast cancer cells. Biochem Biophys Res Commun. 2014;446(2):448-53.

12. Ding J, Lu B, Wang J, Wang J, Shi Y, Lian Y, et al. Long non-coding RNA Loc554202 induces apoptosis in colorectal cancer cells via the caspase cleavage cascades. J Exp Clin Cancer Res. 2015;34(1):100.

13. Nie F-Q, Ma S, Xie M, Liu Y-W, De W, Liu X-H. Decreased long noncoding RNA MIR31HG is correlated with poor prognosis and contributes to cell proliferation in gastric cancer. Tumour Biol. 2016;37(6):7693-701.

14. Yang H, Liu P, Zhang J, Peng X, Lu Z, Yu S, et al. Long noncoding RNA MIR31HG exhibits oncogenic property in pancreatic ductal adenocarcinoma and is negatively regulated by miR-193b. Oncogene. 2016;35(28):3647-57.

15. He A, Chen Z, Mei H, Liu Y. Decreased expression of LnCRNA MIR31HG in human bladder cancer. Cancer Biomark. 2016;17(2):231-6.

16. Chen J, Weiss WA. Alternative splicing in cancer: implications for biology and therapy. Oncogene. 2015;34(1):1-14.

17. Walaszek K, Lower EE, Ziolkowski P, Weber GF. Breast cancer risk in premalignant lesions: osteopontin splice variants indicate prognosis. $\mathrm{Br} J$ Cancer. 2018;119(10):1259-66.

18. Anand V, Khandelwal M, Appunni S, Gupta N, Seth A, Singh P, et al. CD44 splice variant (CD44v3) promotes progression of urothelial carcinoma of bladder through Akt/ERK/STAT3 pathways: novel therapeutic approach. J Cancer Res Clin Oncol. 2019;145(11):2649-61.

19. Yang T, Zhou H, Liu P, Yan L, Yao W, Chen K, et al. IncRNA PVT1 and its splicing variant function as competing endogenous RNA to regulate clear cell renal cell carcinoma progression. Oncotarget. 2017;8(49):85353-67.

20. Paner GP, Stadler WM, Hansel DE, Montironi R, Lin DW, Amin MB. Updates in the eighth edition of the tumor-node-metastasis staging classification for urologic cancers. Eur Urol. 2018;73(4):560-9.

21. Woldu SL, Bagrodia A, Lotan Y. Guideline of guidelines: non-muscle-invasive bladder cancer. BJU Int. 2017;119(3):371-80.
22. Barretina J, Caponigro G, Stransky N, Venkatesan K, Margolin AA, Kim S, et al The Cancer cell line encyclopedia enables predictive modelling of anticancer drug sensitivity. Nature. 2012;483(7391):603-7.

23. Sun W, Duan T, Ye P, Chen K, Zhang G, Lai M, et al. TSVdb: a web-tool for TCGA splicing variants analysis. BMC Genomics. 2018;19(1):405.

24. Lu Q, Ren S, Lu M, Zhang Y, Zhu D, Zhang X, et al. Computational prediction of associations between long non-coding RNAs and proteins. BMC Genomics. 2013;14:651.

25. Schneider CA, Rasband WS, Eliceiri KW. NIH image to ImageJ: 25 years of image analysis. Nat Methods. 2012;9(7):671-5.

26. Gebäck T, Schulz MMP, Koumoutsakos P, Detmar M. TScratch: a novel and simple software tool for automated analysis of monolayer wound healing assays. Biotechniques. 2009:46(4):265-74.

27. Martini T, Heinkele J, Mayr R, Weis CA, Wezel F, Wahby S, et al. Predictive value of lymphangiogenesis and proliferation markers on mRNA level in urothelial carcinoma of the bladder after radical cystectomy. Urol Oncol. 2018:36(12):530.e19-27.

28. Tramm T, Sørensen BS, Overgaard J, Alsner J. Optimal reference genes for normalization of qRT-PCR data from archival formalin-fixed, paraffinembedded breast tumors controlling for tumor cell content and decay of mRNA. Diagn Mol Pathol. 2013;22(3):181-7.

29. Livak KJ, Schmittgen TD. Analysis of relative gene expression data using real-time quantitative $P C R$ and the $2-\Delta \Delta C T$ method. Methods. 2001:25(4): 402-8.

30. Rao X, Huang X, Zhou Z, Lin X. An improvement of the $2^{\wedge}(-$ delta delta CT) method for quantitative real-time polymerase chain reaction data analysis. Biostat Bioinforma Biomath. 2013;3(3):71-85.

31. Zanetti MRD, Petricelli CD, Alexandre SM, Paschoal A, Araujo Júnior E, Nakamura MU. Determination of a cutoff value for pelvic floor distensibility using the Epi-no balloon to predict perineal integrity in vaginal delivery: ROC curve analysis. Prospective observational single cohort study. Sao Paulo Med J. 2016:134(2):97-102

32. Robertson AG, Kim J, Al-Ahmadie H, Bellmunt J, Guo G, Cherniack AD, et al. Comprehensive molecular characterization of muscle-invasive bladder cancer. Cell. 2017;171(3):540-56. e25.

33. Rinaldetti S, Rempel E, Worst TS, Eckstein M, Steidler A, Weiss CA, et al. Subclassification, survival prediction and drug target analyses of chemotherapy-naïve muscle-invasive bladder cancer with a molecular screening. Oncotarget. 2018;9(40):25935-45

34. Network CGAR. Comprehensive molecular characterization of urothelial bladder carcinoma. Nature. 2014;507(7492):315-22.

35. Dandan $W$, Jianliang $C$, Haiyan $H$, Hang M, Xuedong L. Long noncoding RNA MIR31HG is activated by SP1 and promotes cell migration and invasion by sponging miR-214 in NSCLC. Gene. 2019;692:223-30.

36. Shih JW, Chiang WF, Wu ATH, Wu MH, Wang LY, Yu YL, et al. Long noncoding RNA LnCHIFCAR/MIR31HG is a HIF-1a co-activator driving oral cancer progression. Nat Commun. 2017:8:15874.

37. Augoff K, McCue B, Plow EF, Sossey-Alaoui K. miR-31 and its host gene IncRNA LOC554202 are regulated by promoter hypermethylation in triplenegative breast cancer. Mol Cancer. 2012;11:5

38. Stein JP, Lieskovsky G, Cote R, Groshen S, Feng AC, Boyd S, et al. Radical cystectomy in the treatment of invasive bladder cancer: long-term results in 1,054 patients. J Clin Oncol. 2001:19(3):666-75.

39. Rebouissou S, Bernard-Pierrot I, de Reyniès A, Lepage ML, Krucker C, Chapeaublanc $\mathrm{E}$, et al. EGFR as a potential therapeutic target for a subset of muscle-invasive bladder cancers presenting a basal-like phenotype. Sci Transl Med. 2014;6(244):244ra91.

40. Ho PL, Kurtova A, Chan KS. Normal and neoplastic urothelial stem cells: getting to the root of the problem. Nat Rev Urol. 2012;9(10):583-94.

41. McConkey DJ, Choi W, Dinney CP. New insights into subtypes of invasive bladder cancer: considerations of the clinician. Eur Urol. 2014;66(4):609-10.

42. Kardos J, Chai S, Mose LE, Selitsky SR, Krishnan B, Saito R, et al. Claudin-low bladder tumors are immune infiltrated and actively immune suppressed. JCI insight. 2016;1(3):e85902.

43. Sun $Y$, Jia $X$, Wang M, Deng $Y$. Long noncoding RNA MIR31HG abrogates the availability of tumor suppressor microRNA-361 for the growth of osteosarcoma. Cancer Manag Res. 2019;11:8055-64.

44. Yan S, Tang Z, Chen K, Liu Y, Yu G, Chen Q, et al. Long noncoding RNA MIR31HG inhibits hepatocellular carcinoma proliferation and metastasis by sponging microRNA-575 to modulate ST7L expression. J Exp Clin Cancer Res. 2018:37(1):214. 
45. Sun K, Zhao X, Wan J, Yang L, Chu J, Dong S, et al. The diagnostic value of long non-coding RNA MIR31HG and its role in esophageal squamous cell carcinoma. Life Sci. 2018;202:124-30.

46. Hu R, Lu C, Mostaghel EA, Yegnasubramanian S, Gurel M, Tannahill C, et al. Distinct transcriptional programs mediated by the ligand-dependent fulllength androgen receptor and its splice variants in castration-resistant prostate cancer. Cancer Res. 2012;72(14):3457-62.

47. Sun S, Sprenger CC, Vessella RL, Haugk K, Soriano K, Mostaghel EA, et al. Castration resistance in human prostate cancer is conferred by a frequently occurring androgen receptor splice variant. J Clin Invest. 2010;120(8):2715-30.

48. Poulikakos PI, Persaud Y, Janakiraman M, Kong X, Ng C, Moriceau G, et al. RAF inhibitor resistance is mediated by dimerization of aberrantly spliced BRAF(V600E). Nature. 2011;480(7377):387-90.

49. Salton M, Kasprzak WK, Voss T, Shapiro BA, Poulikakos PI, Misteli T. Inhibition of vemurafenib-resistant melanoma by interference with pre-mRNA splicing. Nat Commun. 2015;6:7103

50. Chaux A, Cohen JS, Schultz L, Albadine R, Jadallah S, Murphy KM, et al. High epidermal growth factor receptor immunohistochemical expression in urothelial carcinoma of the bladder is not associated with EGFR mutations in exons 19 and 21: a study using formalin-fixed, paraffin-embedded archival tissues. Hum Pathol. 2012;43(10):1590-5.

51. Wang B, Jiang H, Wang L, Chen X, Wu K, Zhang S, et al. Increased MIR31HG IncRNA expression increases gefitinib resistance in non-small cell lung cancer cell lines through the EGFR/PI3K/AKT signaling pathway. Oncol Lett. 2017;13(5):3494-500

\section{Publisher's Note}

Springer Nature remains neutral with regard to jurisdictional claims in published maps and institutional affiliations.

Ready to submit your research? Choose BMC and benefit from:

- fast, convenient online submission

- thorough peer review by experienced researchers in your field

- rapid publication on acceptance

- support for research data, including large and complex data types

- gold Open Access which fosters wider collaboration and increased citations

- maximum visibility for your research: over $100 \mathrm{M}$ website views per year

At $\mathrm{BMC}$, research is always in progress.

Learn more biomedcentral.com/submissions 